\title{
SERUM PROTEASES DO NOT CLEAVE PROLACTIN TO VASOINHIBINS AT PHYSIOLOGICAL pH
}

Jakob Triebel ${ }^{1}$, Nicole Schauer ${ }^{1,2}$, Felix Hesslinger ${ }^{1}$, Aura Ileana Moreno-Vega ${ }^{3}$, Gonzalo Martínez de la Escalera ${ }^{3}$, Carmen Clapp $^{3}$, and Thomas Bertsch ${ }^{1}$

${ }^{1}$ Institute for Clinical Chemistry, Laboratory Medicine and Transfusion Medicine, Paracelsus Medical University, Nuremberg, Germany

${ }^{2}$ Georg Simon Ohm University Nuremberg, Nuremberg, Germany

${ }^{3}$ Instituto de Neurobiología, Universidad Nacional Autónoma de México (UNAM), Campus UNAM-Juriquilla, Querétaro, México

\section{BACKGROUND}

The prolactin/vasoinhibin axis constitutes a novel endocrine axis in which the generation, secretion, and actions of the pituitary hormones prolactin and vasoinhibins are under control of the hypothalamus, the pituitary gland, and the target tissue microenvironment. Vasoinhibins are generated in the pituitary gland and in multiple target tissues through proteolytic cleavage of prolactin. Here, we investigated whether prolactin is cleaved to vasoinhibins in the circulation.

\section{The prolactin/vasoinhibin axis essentials}

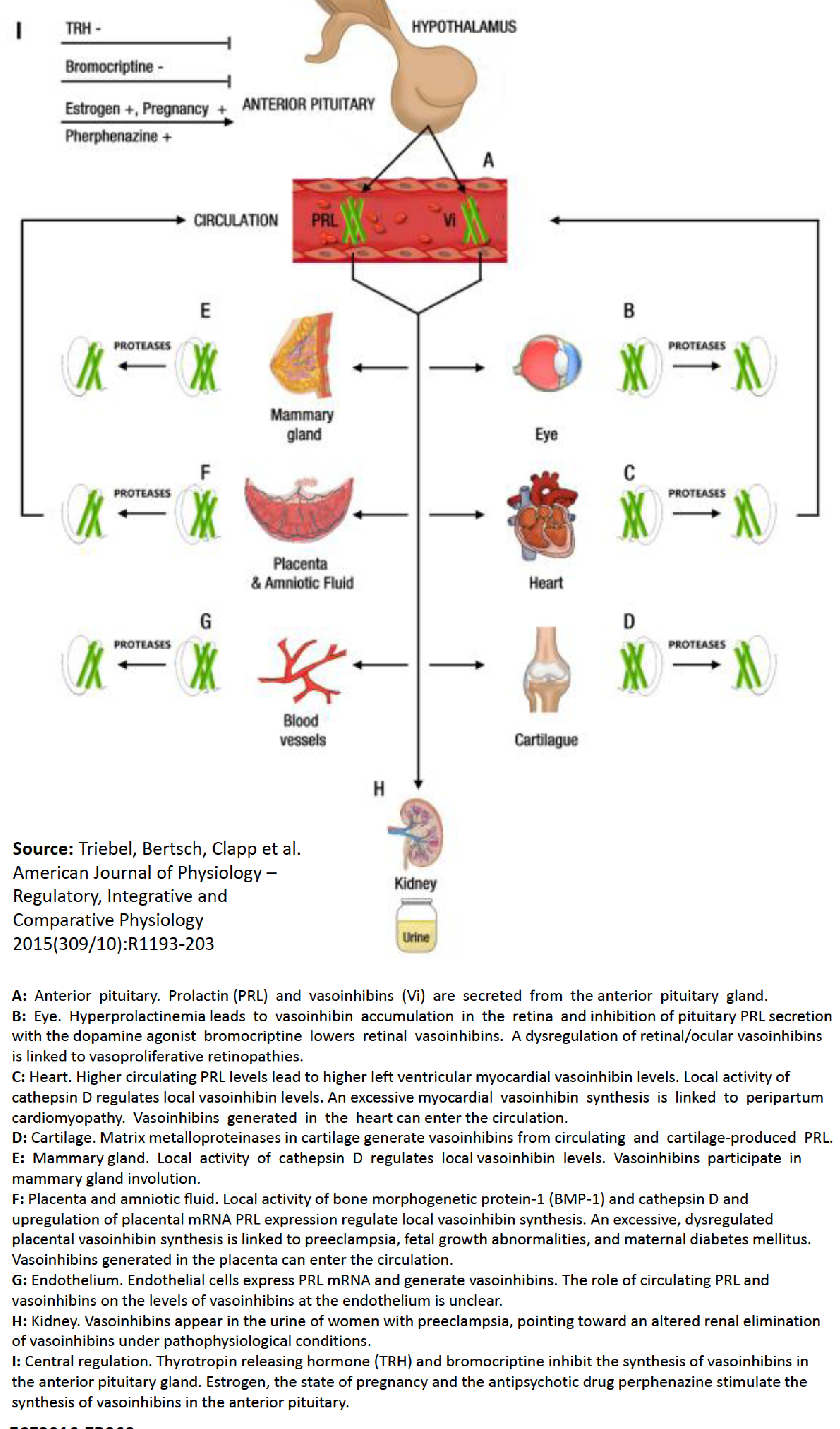

\section{METHODS}

Human serum or plasma and recombinant human prolactin were incubated at $37^{\circ} \mathrm{C}$ alone and in combination, with a variation of $\mathrm{pH}$, time, and buffer composition. The samples were resolved on sodium dodecyl sulfate polyacrylamide gel electrophoresis and Western Blotting was performed using anti-prolactin and antivasoinhibin antibodies.

\section{RESULTS}

Sodium dodecyl sulfate polyacrylamide gel electrophoresis and Western Blotting analyses demonstrated the presence of the recombinant prolactin-protein with an apparent molecular mass of $23 \mathrm{kDa}$. Samples incubated throughout a time period ranging from 10 minutes to 24 hours at $\mathrm{pH} 7.4$ did not demonstrate immunoreactive bands lower than $23 \mathrm{kDa}$. An anti-vasoinhibin immunoreactive band featuring an apparent molecular mass of $17 \mathrm{kDa}$ was intermittently observed in samples incubated at $\mathrm{pH} 3.4$.

\section{Gelelectrophoresis \& Western Blotting}

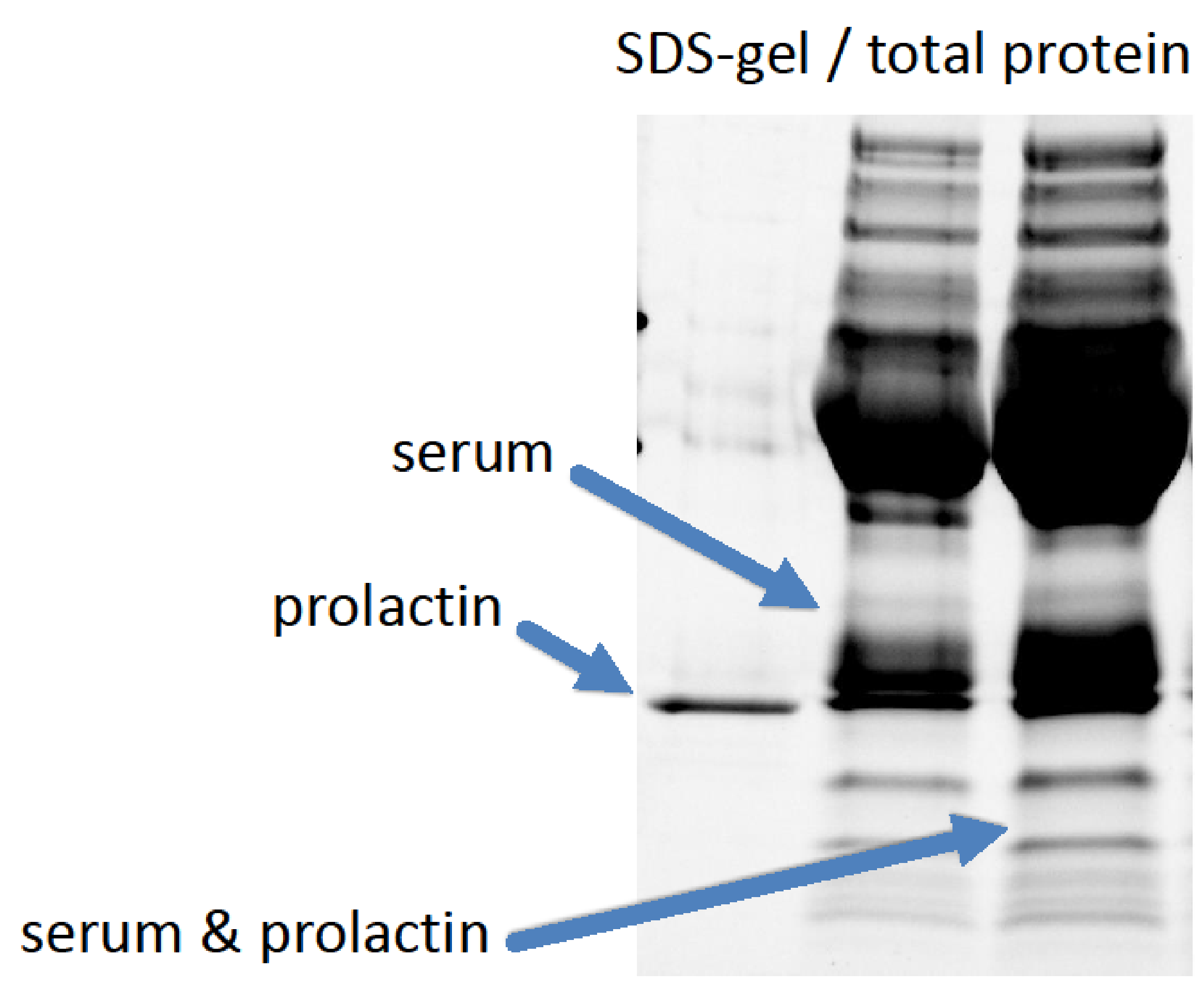

PVDF / anti-prolactin antibody multichannel

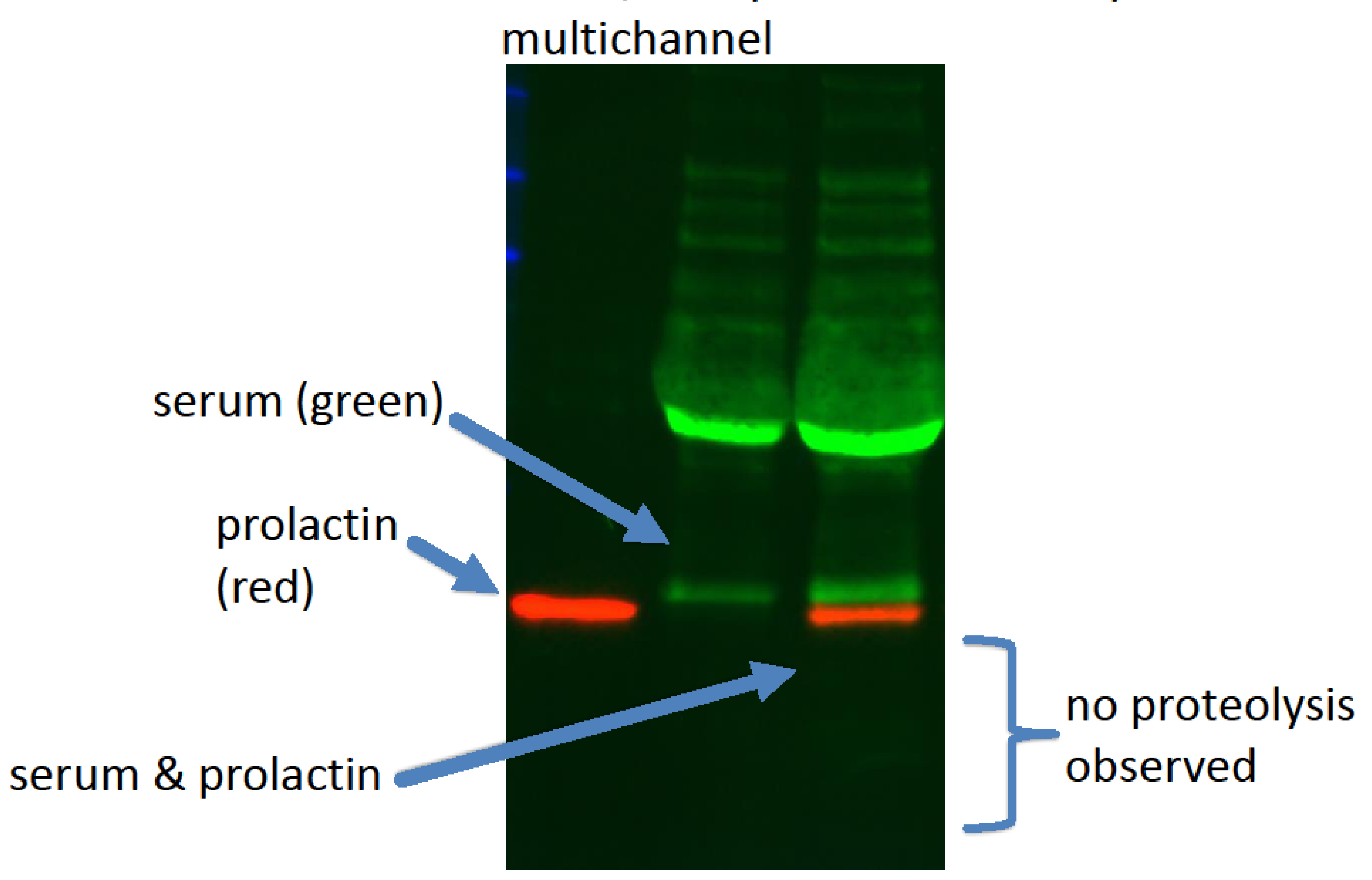

PVDF / anti-prolactin antibody chemiluminescence

prolactin

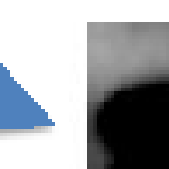

proteolytic prolactin fragment $17 \mathrm{kDa}$

\section{CONCLUSION}

Vasoinhibin generation by enzymatic cleavage of prolactin does not occur in human serum under physiological conditions. The generation of vasoinhibins seems to be restricted to the pituitary gland and the target tissue level. A limited proteolysis of prolactin, resulting in the generation of a prolactin-fragment with an apparent molecular weight of $17 \mathrm{kDa}$, seems to occur at acid $\mathrm{pH}$. 